\title{
HIGHER DERIVATIONS ON FINITELY GENERATED INTEGRAL DOMAINS. II
}

\author{
WILLIAM C. BROWN
}

ABSTRACT. We prove

Theorem. Let $A=k\left[x_{1}, \ldots, x_{m}\right]$ be a finitely generated integral domain over a field $k$ of characteristic zero. Then $A$ regular, i.e. the local ring $A_{q}$ is regular for every prime ideal $q \subseteq A$, is equivalent to the following two conditions: (1) no prime of $A$ of height greater than one is differential, and (2) for all $\phi \in \operatorname{Hom}_{k}(A, A), \phi \in \operatorname{Der}_{k}^{n}(A)$ if and only if $\Delta \phi \in \mathbf{\Sigma}_{i=1}^{n-1} \operatorname{Der}_{k}^{i}(A) \cup \operatorname{Der}_{k}^{n-i}(A)(n=1,2, \ldots)$.

Here $\Delta$ denotes the Hochschild coboundary operator, $U$ denotes the cup product, and $\operatorname{Der}_{k}^{n}(R)$ is the module of higher derivations of rank $n$.

Introduction. Throughout this paper, $A=k\left[x_{1}, \ldots, x_{m}\right]$ will denote a finitely generated integral domain over a field $k$ of characteristic zero. For each $n=1,2, \ldots:$, we shall let $\operatorname{Der}_{k}^{n}(A)$ denote the $A$-module of all $n$th order derivations of $A$ to $A$ which vanish on $k$. Thus, $\phi \in \operatorname{Der}_{k}^{n}(A)$ if and only if $\phi \in \operatorname{Hom}_{k}(A, A)$, and for all $a_{0}, \ldots, a_{n} \in A$ we have

$$
\begin{aligned}
& \phi\left(a_{0} a_{1} \cdots a_{n}\right) \\
& \quad=\sum_{s=1}^{n}(-1)^{s-1} \sum_{i_{1} \cdots i_{s}} a_{i_{1}} \cdots a_{i_{s}} \phi\left(a_{0} \cdots \check{a}_{i_{1}} \cdots \check{a}_{i_{s}} \cdots a_{n}\right) .
\end{aligned}
$$

The author refers the reader to [5] for the various properties of the modules $\operatorname{Der}_{k}^{n}(A)$ which are used in this paper.

It follows from [5, Proposition 4 and Corollary 6.1] that any composite $\delta_{1} \ldots \delta_{j}(1 \leq j \leq n)$ of $j$-derivations $\delta_{i} \in \operatorname{Der}_{k}^{1}(A)$ is an $n$th order derivation. The $A$-submodule of $\operatorname{Der}_{k}^{n}(A)$ spanned by all such composites will be denoted by $\operatorname{der}_{k}^{n}(A)$.

We shall say that a prime ideal $p$ in $A$ is differential if $\delta(p) \subseteq p$ for all $\delta \in \operatorname{Der}_{k}^{1}(A)$. We shall call $A$ a regular ring if for all prime ideals $q \subseteq A$,

Received by the editors February 28, 1974.

AMS (MOS) subject classifications (1970). Primary $13 \mathrm{H} 05$.

Key words and phrases. $n$th order derivation, $\operatorname{der}_{k}^{n}(A)$. 
the local ring $A_{q}$ is regular. With these definitions, the author proved [1]

Theorem 1. Let $A=k\left[x_{1}, \ldots, x_{m}\right]$ be a finitely generated integral domain over a field $k$ of characteristic zero. Then $A$ is a regular ring if and only if the following two properties are satisfied: (a) no prime $p$ of $A$ of height greater than one is differential and (b) $\operatorname{Der}_{k}^{n}(A)=\operatorname{der}_{k}^{n}(A)$ for all $n$.

Thus, the arithmetic condition (b) on the modules $\operatorname{Der}_{k}^{n}(A)$ in the presence of (a) implies regularity. The purpose of this paper is to show that the condition (b) can be replaced by another quite different arithmetic condition on the modules $\operatorname{Der}_{k}^{n}(A)$.

Recall that if $\phi$ and $\psi$ are $k$-linear mappings of $A$ into itself, then the Hochschild coboundary $\Delta \phi$ of $\phi$ is the $k$-bilinear mapping $\Delta \phi: A \times A \rightarrow A$ given by $\Delta \phi(a, b)=\phi(a b)-a \phi(b)-b \phi(a)$ for all $a, b \in A$. The cup product $\phi \cup \psi: A \times A \rightarrow A$ is the $k$-bilinear mapping defined by $\phi \cup \psi(a, b)=$ $\phi(a) \psi(b)$. If $P$ and $Q$ are two $A$-submodules of $\operatorname{Hom}_{k}(A, A)$, then $P \cup Q$ will denote the set of all $k$-bilinear mappings of $A \times A \rightarrow A$ which are finite sums of mappings of the form $\phi \cup \psi$ for $\phi \in P, \psi \in Q$.

Let us set

$$
\sum(A)=\sum_{i=1}^{n-1} \operatorname{Der}_{k}^{i}(A) \cup \operatorname{Der}_{k}^{n-i}(A) .
$$

Then it follows from [5, Proposition 3] that $\phi \in \operatorname{Der}_{k}^{n}(A)$ if $\Delta \phi \in \Sigma(A)$. In [3], Y. Ishibashi proved

Theorem 2. Let $A$ be a finitely generated algebra over a field $k$ such that $\Omega_{k}^{i}(A)$ is $A$-projective for every $i \geq 1$. Let $\phi \in \operatorname{Hom}_{k}(A, A)$. Then $\phi \in \operatorname{Der}_{k}^{n}(A)$ if and only if $\Delta \phi \in \Sigma(A)$. Here $\Omega_{k}^{i}(A)$ denotes the A-module of ith order differentials.

If $A$ is a finitely generated integral domain over a field $k$ of characteristic zero, then we shall prove a partial converse to Theorem 2.

Main results.

Theorem 3. Let $A=k\left[x_{1}, \ldots, x_{m}\right]$ be a finitely generated integral domain over a field $k$ of characteristic zero. Then $A$ is a regular ring if and only if the following two conditions are satisfied:

(a) no prime ideal $p$ of $A$ of height greater than one is differential; and

(b) for every positive integer $n$ and every $\phi \in \operatorname{Hom}_{k}(A, A) \phi \epsilon$ $\operatorname{Der}_{k}^{n}(A)$ if and only if $\Delta \phi \in \Sigma(A)$.

Proof. Suppose $A$ is a regular ring. Then by Theorem $1, A$ satisfies 
(a) and for each positive integer $n$, $\operatorname{Der}_{k}^{n}(A)=\operatorname{der}_{k}^{n}(A)$. But if every $\phi \epsilon$ $\operatorname{Der}_{k}^{n}(A)$ is a linear combination of composites of first order derivations, then a simple induction argument shows $\Delta \phi \in \Sigma(A)$. Thus, one direction of Theorem 3 follows immediately from Theorem 1 .

Now suppose $A$ satisfies conditions (a) and (b). We shall first show that $A$ is integrally closed. Let $Q$ denote the quotient field of $A$, and let $\bar{A}$ denote the integral closure of $A$ in $Q$. Let us assume that $A \neq \bar{A}$. Then the conductor $C$ of $A$ is a proper ideal in $A$. By [6, Corollary, p. 169], $C$ is a differential ideal. Let $p$ be an associated prime of $C$. Then by $[7$, Theorem 1], $p$ is differential, and thus by condition (a) $p$ is a minimal prime of $A$.

Now let $R=A_{\underline{p}}$ (A localized at $p$ ). Then $\bar{R}$, the integral closure of $R$ in $Q$, is given by $\bar{A}_{p}$. The conductor of $R$ in $\bar{R}$ is just $C R$. We note that $\bar{R}$ is a semilocal ring with maximal ideals say $p_{1}, \ldots, p_{s}$. If we set $V_{i}=$ $\bar{R}_{p_{i}}, i=1, \ldots, s$, then $V_{i}$ is a discrete rank one valuation ring, and $\bar{R}=$ $\bigcap_{i=1}^{p_{i}} V_{i}$. Since $\Omega_{k}^{n}(R)=\Omega_{k}^{n}(A) \otimes_{A} A_{p}$ [5, Theorem 9], and $A_{p}$ is a flat $A$ module, we see that $\operatorname{Der}_{k}^{n}(R)=\operatorname{Der}_{k}^{n}(A) \otimes_{A} A_{p}$. So any derivation $\phi$ in $\operatorname{Der}_{k}^{n}(R)$ has the form $(1 / s) \phi^{\prime}$ with $\phi^{\prime} \in \operatorname{Der}_{k}^{n}(A), s$ an element of $A$ not in $p$. Thus, since $A$ satisfies (b), one easily sees that if $\phi \in \operatorname{Der}_{k}^{n}(R)$, then $\Delta \phi \in \Sigma(R)$. On the other hand, if $\phi \in \operatorname{Hom}_{k}(R, R)$, and $\Delta \phi \in \Sigma(R)$, then it follows from [5, Proposition 3] that $\phi \in \operatorname{Der}_{k}^{n}(R)$. Thus, $R$ satisfies condition (b) in the theorem.

Let the tanscendence degree of $Q$ over $k$ be $r$, and let $\Omega_{k}^{1}(T)$ denote the module of first order differentials of any $k$-algebra $T$. It follows from [4, Theorem $\left.3^{\prime}\right]$ that $\Omega_{k}^{1}\left(V_{i}\right)$ is a free $V_{i}$-module necessarily of rank $r$. Since $\bar{R}$ is semilocal, it follows that $\operatorname{Der}_{k}^{1}(\bar{R})$ is a free $\bar{R}$-module of rank $r$. Let $\bar{d}: \bar{R} \rightarrow \Omega_{k}^{1}(\bar{R})$ denote the canonical derivation of $\bar{R}$ into $\Omega_{k}^{1}(\bar{R})$.

Since the depth of $p$ is $r-1$, the quotient field of $A / p$ has transcendence degree $r-1$ over $k$. Hence there exist elements $\alpha_{1}, \ldots, a_{r-1} \epsilon$ $A-p$ such that the quotient field of $A / p$ is a separable algebraic extension of $k\left(\bar{\alpha}_{1}, \ldots, \bar{\alpha}_{r-1}\right)$. Here $\bar{\alpha}_{i}$ of course denotes the image of $\alpha_{i}$ in $A / p$. Note that the field $F=k\left(\alpha_{1}, \ldots, \alpha_{r-1}\right)$ is contained in $R$.

Using [8, Theorem 18, p. 45], we can find an element $\beta \in \bigcap_{i=1}^{s} p_{i}$ such that $\beta$ generates the maximal ideal in each local ring $V_{i}, i=1, \ldots, s$. It now follows from the proof of [4, Theorem $\left.3^{\prime}\right]$ and Nakayama's lemma that $\left\{\bar{d}(\beta), \bar{d}\left(\alpha_{1}\right), \ldots, \bar{d}\left(\alpha_{r-1}\right)\right\}$ is a free $\bar{R}$-module basis of $\Omega_{k}^{1}(\bar{R})$. Since $\operatorname{Hom}_{R}\left(\Omega_{k}^{1}(\bar{R}), \bar{R}\right) \cong \operatorname{Der}_{k}^{r}(\bar{R})$, there exist derivations $\delta_{0}, \delta_{1}, \ldots, \delta_{r-1} \epsilon$ $\operatorname{Der}_{k}^{1}(\bar{R})$ such that the following equations are satisfied: 


$$
\delta_{0}(\beta)=1, \quad \delta_{0}\left(\alpha_{i}\right)=0=\delta_{i}(\beta), \quad i=1, \ldots, r-1,
$$

and

$$
\delta_{i}\left(a_{j}\right)= \begin{cases}0 & \text { if } i \neq j, i, j=1, \ldots, r-1, \\ 1 & \text { if } i=j .\end{cases}
$$

Clearly $\left\{\delta_{0}, \ldots, \delta_{r-1}\right\}$ is a free $\bar{R}$-module basis of $\operatorname{Der}_{k}^{1}(\bar{R})$ and also a $Q$ algebra basis for $\bigcup_{n=1}^{\infty} \operatorname{Der}_{k}^{n}(Q)$.

If $\phi \in \operatorname{Der}_{k}^{n}(R)$, then by [5, Theorem 15] $\phi \in \operatorname{Der}_{k}^{n}(Q)$. Thus, $\phi$ can be written uniquely as a polynomial (with coefficients in $Q$ ) in $\delta_{0}, \ldots, \delta_{r-1}$ of degree less than or equal to $n$. Let $J=\bigcap_{i=1}^{s} p_{i}$, the Jacobson radical of $\bar{R}$. We need the following

Lemma. Let $\phi \in \operatorname{Der}_{k}^{n}(R)$. Then $\phi=g\left(\delta_{0}, \ldots, \delta_{r-1}\right)$, where $g\left(X_{0}, \ldots, X_{r-1}\right)$ is a polynomial in indeterminates $X_{i}$ with coefficients in $Q$. Furthermore, the coefficient of $\delta_{0}^{n}$ in $g$ is an element of $J^{n}$.

Proof. Only the last statement in the Lemma needs proof. We proceed via induction on $n$. Suppose $\phi \in \operatorname{Der}_{k}^{1}(R)$. By [6, Corollary, p. 169], $C R$ is differential under $\phi$. Since $\phi \in \operatorname{Der}_{k}^{1}(\bar{R}), C \bar{R}$ is differential under $\phi$. Thus, every associated prime of $C \bar{R}$ is differential under $\phi$. Since every $p_{i}$ is an associated prime of $C \bar{R}$, we have $\phi\left(p_{i}\right) \subseteq p_{i}, i=1, \ldots, s$. Thus, $\phi(\beta) \in J$. But if $\phi=\Sigma_{i=0}^{r-1} a_{i} \delta_{i}, a_{i} \in Q$, then $\phi(\beta)=a_{0}$. Thus the Lemma is proven in the case $n=1$.

We shall sketch the proof of the $n=2$ case before proceeding to the inductive step. Suppose $\phi \in \operatorname{Der}_{k}^{2}(R)$. Since $R$ satisfies condition (b), there exist $\lambda_{l}, \psi_{l} \in \operatorname{Der}_{k}^{1}(R)$, and elements $e_{l} \in R$ such that for all $a$ and $b$ in $R$ we have

$$
\phi(a b)=a \phi(b)+b \phi(a)+\sum_{l} e_{l} \psi_{l}(a) \lambda_{l}(b) .
$$

One easily checks using [5, Theorem 15] that (3) holds for all $a, b \in Q$. Now write

$$
\phi=\sum_{i=0}^{r-1} a_{i} \delta_{i}+\sum_{i_{1}, i_{2}=0}^{r-1} a_{i_{1} i_{2}} \delta_{i_{1}} \delta_{i_{2}} \text { with } a_{i^{\prime}}, a_{i_{1} i_{2}} \in Q
$$

and

$$
\psi_{l}=\sum_{i=0}^{r-1} \mu_{i l} \delta_{i}, \quad \lambda_{l}=\sum_{i=0}^{r-1} \gamma_{i l} \delta_{i} \text { with } \mu_{i l}, \gamma_{i l} \in Q .
$$

From the case $n=1$, we have $\mu_{0 l}, \gamma_{0 l} \in J$. Now substitute (4) and (5) into (3) and obtain 


$$
\begin{aligned}
\sum_{i_{1}, i_{2}} a_{i_{1} i_{2}}\left(\delta_{i_{1}}(a) \delta_{i_{2}}(b)+\delta_{i_{1}}(b) \delta_{i_{2}}(a)\right) \\
=\sum_{l} e_{l}\left(\sum_{i=0}^{r-1} \mu_{i l} \delta_{i}(a)\right)\left(\sum_{i=0}^{r-1} \gamma_{i l} \delta_{i}(b)\right) .
\end{aligned}
$$

If we now substitute $a=b=\beta$ in (6), we get $2 a_{00}=\Sigma_{l} e_{l} \mu_{0 l} \gamma_{01} \in J^{2}$. Thus $a_{00} \in J^{2}$ and the case $n=2$ is complete.

Assume we have now proven the Lemma for $t=1,2, \ldots, n-1(n \geq 3)$, and consider $\phi \in \operatorname{Der}_{k}^{n}(R)$. Then by condition (b) there exist elements $e_{l, j} \in R$ and derivations $\psi_{l}^{(j)}, \lambda_{l}^{(j)} \in \operatorname{Der}_{k}^{j}(R), j=1, \ldots, n-1$, such that for all $a, b \in R$ we have

$$
\begin{aligned}
\phi(a b)= & a \phi(b)+b \phi(a)+\sum e_{l 1} \psi_{l}^{(1)}(a) \lambda_{l}^{(n-1)}(b) \\
& +\sum_{l} e_{l 2} \psi_{l}^{(2)}(a) \lambda_{l}^{(n-2)}(b)+\cdots+\sum_{l} e_{l n-1} \psi_{l}^{(n-1)}(a) \lambda_{l}^{(1)}(b) .
\end{aligned}
$$

Now write $\phi$ as a polynomial $g\left(\delta_{0}, \ldots, \delta_{r-1}\right)$ of degree less than or equal to $n$. If the coefficient of $\delta_{0}^{n}$ in $g$ is zero, then we have nothing to prove. Thus, without loss of generality, we can assume that the coefficient of $\delta_{0}^{n}$ in $g$ is not zero. By the induction hypothesis, we can write each $\psi_{l}^{(j)}$ and $\lambda_{l}^{(j)}$ as a polynomial in $\delta_{0}, \delta_{1}, \ldots, \delta_{r-1}$ whose coefficient of $\delta_{0}^{j}$ is in $J^{j}$. Say

$$
\psi_{l}^{(j)}=\sum c_{t}^{l, j} \delta_{t}+\sum c_{t_{1} t_{2}{ }^{l}{ }_{1}{ }_{1}{ }_{t_{2}}}^{l, j} \delta+\sum c_{t_{1} \cdots t_{j}}^{l, j} \delta_{t_{1}} \cdots \delta_{t_{j}}
$$

and

$$
\lambda_{l}^{(j)}=\sum b_{t}^{l, j} \delta_{t}+\sum b_{t_{1} t_{2} t_{1}}^{l, j} \delta_{t_{2}}+\cdots+\sum b_{t_{1} \cdots t_{j}{ }_{t_{1}}}^{l, j} \delta_{t_{j}}
$$

We now substitute $\phi=g\left(\delta_{0}, \ldots, \delta_{r-1}\right)$ and the expressions in (8) into (7). Using the relations in (2), we can eliminate all terms in (7) which involve any $\delta_{i}, i=1, \ldots, r-1$. Thus, (7) reduces to an equation of the form

$$
\begin{aligned}
\sum_{i=1}^{n} a_{i} \delta_{0}^{i}(a b)= & a\left(\sum_{i=1}^{n} a_{i} \delta_{0}^{i}(b)\right)+b\left(\sum_{i=1}^{n} a_{i} \delta_{0}^{i}(a)\right) \\
& +\sum_{l} e_{l 1}\left(c_{0}^{l, 1} \delta_{0}(a)\right)\left(b_{0}^{l, n-1} \delta_{0}+\cdots+b_{0 \cdots 0}^{l, n-1} \delta_{0}^{n-1}\right)(b) \\
& +\cdots+\sum_{l} e_{l, n-1}\left(c_{0}^{l, n-1} \delta_{0}+\cdots+c_{0 \cdots 0}^{l, n-1} \delta_{0}^{n-1}\right)(a)\left(b_{0}^{l, 1} \delta_{0}(b)\right)
\end{aligned}
$$


Here $c_{0 \ldots 0}^{l, j}, b_{0 \ldots 0}^{l, j} \in J^{j}$ for each $j=1, \ldots, n-1$, when the number of zeros appearing in the subscript is $j$.

If we now make various substitutions of the form $a=\beta^{s}, b=\beta^{t}$ and eliminate sums which are equal, we easily see that $a_{n} \in J^{n}$. Since $a_{n}$ is the coefficient of $\delta_{0}^{n}$ appearing in $g$, the proof is complete.

Using the Lemma, we can easily argue that $A$ is integrally closed. Let $c \in C R$. Then $c \delta_{0}^{n} \in \operatorname{Der}_{k}^{n}(R)$ for every $n$. Thus by the Lemma, $c \in J^{n}$. Since $c$ is arbitrary, we have $C R \subset \bigcap_{n=1}^{\infty} J^{n}$. Since $J$ is the Jacobson radical of $\bar{R}$, we have $\bigcap_{n=1}^{\infty} J^{n}=0$. Thus $C R=(0)$. But we are assuming $A \neq \bar{A}$, and thus $C R \neq(0)$, a contradiction. Thus, we have shown that conditions (a) and (b) imply that $A$ is integrally closed.

We now show that $A$ is a regular ring. Let $q$ be a minimal prime of $A$. Then $A_{q}$ is a discrete rank one valuation ring. In particular, $A_{q}$ is a regular local ring. Assume we have shown that $A_{q}$ is a regular local ring for all primes $q$ of height less than or equal to $t$. Let $q$ be a prime of height $t+1$. Then by (a) $q$ is not differential under $\operatorname{Der}_{k}^{1}(A)$. Then $q A_{q}$ is not differential under $\operatorname{Der}_{k}^{1}\left(A_{q}\right)$. We note that the induction hypothesis implies that every proper localization of $A_{q}$ is regular. It now follows from [7, Theorem 5] that $A_{q}$ is regular. This completes the proof of Theorem 3.

It follows from $[2,16.12 .12]$ that if $A$ is regular, then $A$ is a smooth algebra over $k$. Thus Theorem 3 can be viewed as a partial converse of Theorem 2 .

Example. In studying the proofs of Theorems 1 and 3, we see that the following theorem proven by A. Seidenberg [6] is of crucial importance.

Theorem 4. Let $R$ be an integral domain containing the rational numbers $k$, and let $R^{\prime}$ be the ring of elements in the quotient field of $R$ which are quasi-integral aver $R$. Let $\delta \in \operatorname{Der}_{k}^{1}(R)$. Then $\delta \in \operatorname{Der}_{k}^{1}\left(R^{\prime}\right)$.

It is well known that this theorem is false if $k$ is a field of characteristic not zero. It is somewhat surprising that this theorem is also false (in the characteristic zero case) for higher derivations. We give an example.

Consider the curve $x^{2}=y^{3}$ over the rational numbers $k$. That is, let $A=k[X, Y] /\left(X^{2}-Y^{3}\right)=k[x, y]$. One easily checks that $A$ is an integral domain whose integral closure $\bar{A}$ in the quotient field $Q$ of $A$ is given by $\bar{A}=A[x / y]$. Since $Q$ is a separable algebraic extension of $k(y)$, it follows that any $\phi \in \operatorname{Der}_{k}^{2}(Q)$ is uniquely determined by its values on $y$ and $y^{2}$. A simple calculation shows that if $\phi(y)=a$ and $\phi\left(y^{2}\right)=b$, then

$$
\phi(x)=\frac{3 y}{8}\left(\frac{2 y a+b}{x}\right), \quad \phi\left(x^{2}\right)=3 y b-3 y^{2} a \quad \text { and } \quad \phi(x y)=\frac{5 y^{2}}{8}\left(\frac{3 b-2 y a}{x}\right) \text {. }
$$


In particular, if we set $a=1$ and $b=-2 y$, then $\phi \in \operatorname{Der}_{k}^{2}(A)$ and is given by

(10) $\phi(y)=1, \quad \phi\left(y^{2}\right)=-2 y, \quad \phi(x)=0, \quad \phi\left(x^{2}\right)=-9 y^{2} \quad$ and $\quad \phi(x y)=-5 x$.

The conductor $C$ of $A$ is given by $C=(x, y)$. Note that $\phi(C) \not \subset C$. Using [5, Theorem 15], we see that $\phi(x / y)=x / y^{2} \notin \bar{A}$. Thus, higher derivations on $A$ need not map $\bar{A}$ into $\bar{A}$.

Finally the author notes that in the above example (and all other examples investigated so far) if $\phi \in \operatorname{Der}_{k}^{2}(A)$ satisfies $\Delta \phi \in \operatorname{Der}_{k}^{1}(A) \cup \operatorname{Der}_{k}^{1}(A)$, then $\phi(\bar{A}) \subset \bar{A}$. This leads to the following conjecture. Suppose $A$ is a finitely generated integral domain over a field $k$ of characteristic zero. Let $\phi \epsilon$ $\operatorname{Der}_{k}^{n}(A)$, and suppose $\Delta \phi \in \Sigma(A)$. Suppose further that the derivations $\psi_{l}^{(i)}$, $\lambda_{l}^{(j)}$ (as in (7)) used in $\Delta \phi$ map the integral closure $\bar{A}$ of $A$ into itself. Then $\phi$ maps $\bar{A}$ to $\bar{A}$.

\section{REFERENCES}

1. W. C. Brown, Higher derivations on finitely generated integral domains, Proc. Amer. Math. Soc. 42 (1974), 23-27.

2. A. Grothendieck, Éléments de géométrie algébrique, IV, Étude locale des schémas et des morphismes de schémas. IV, Inst. Hautes Études Sci. Publ. Math. No. 32, (1967). MR 39 \# 220.

3. Y. Ishibashi, Some remarks on high order derivations, Pacific J. Math. 52 (1974), 419-424.

4. Y. Nakai, On the theory of differentials in commutative rings, J. Math. Soc. Japan 13 (1961), 63-84. MR 23 \# A2437.

5. — - Higher order derivations. I, Osaka J. Math. 7 (1970), 1-27. MR $41 \# 8404$.

6. A. Seidenberg, Derivations and integral closure, Pacific J. Math. 16 (1966), 167-173. MR 32 \# 5686 .

7. - Differential ideals in rings of finitely generated type, Amer. J. Math. 89 (1967), 22-42. MR 35 \# 2902.

8. O. Zariski and P. Samuel, Commutative algebra. Vol. II, University Series in Highter Math., Van Nostrand, Princeton, N. J., 1960. MR 22 \# 11006.

DEPARTMENT OF MATHEMATICS, MICHIGAN STATE UNIVERSITY, EAST LANSING, MICHIGAN 48824 